\title{
The relationship between prenatal testosterone and developmental stuttering in boys
}

\author{
Yunus Emre Dönmez¹, Özlem Özcan², Ayhan Bilgiç³, Dilşad Yıldız Miniksar ${ }^{1}$ \\ ${ }^{1}$ Department of Child and Adolescent Psychiatry, Malatya Training and Research Hospital; ${ }^{2}$ Department of Child and \\ Adolescent Psychiatry, Inönü University Faculty of Medicine, Malatya; ${ }^{3}$ Department of Child and Adolescent Psychiatry, \\ Necmettin Erbakan University, Meram Faculty of Medicine, Konya, Turkey. E-mail: dryemredonmez@gmail.com \\ Received: 1st May 2018, Revised: 1st July 2018, Accepted: 10th July 2018
}

SUMMARY: Dönmez YE, Özcan Ö, Bilgiç A, Miniksar DY. The relationship between prenatal testosterone and developmental stuttering in boys. Turk J Pediatr 2019; 61: 193-199.

Stuttering is a multi-factorial speech disorder characterized by various dysfluencies in the beginning and/or smooth flow of speech. Its incidence is about $1 \%$ in the general population, with $80 \%$ of stuttering cases seen in children aged three to eight years. The etiology of developmental stuttering has not been precisely explained. Several researchers report that neurological factors, especially atypical lateralization of the speech and language processes, play an important role in the etiology of stuttering. Various hypotheses suggest that prenatal testosterone causes atypical hemispheric lateralization. The aim of this study was to investigate the role of prenatal testosterone in the etiology of developmental stuttering by the use of the ratio of secondand fourth-digit lengths (2D:4D).

Totally 133 boys (49 boys who stutter and 84 boys who do not stutter) were included in the study. The age range of children participating in the study was determined as $2-12$ years. In the comparative evaluation conducted between the stuttering group and the control group in terms of their right 2D:4D and left 2D:4D ratios, it was found that the left 2D:4D ratio of the patients was significantly higher than that of the control group.

We are of the opinion that prenatal testosterone may play a role in the etiology of developmental stuttering. The present study is the first study in the literature to reveal a relationship between prenatal testosterone and developmental stuttering.

Key words: stuttering, testosterone, childhood, boys, callosal hypothesis.

Stuttering is a multi-factorial speech disorder characterized by the repetition of sounds, syllables, and words resulting in the prolongation or blocking of sounds or syllables at the beginning of speech or interrupting its smooth flow. ${ }^{1}$ Its incidence is about $1 \%$ in the general population with $80 \%$ of stuttering occurring in children aged three to eight years. The incidence of stuttering in preschool and school-age children is about $4 \% .^{1,2}$ Stuttering is more common in males than in females in all age groups. ${ }^{3}$ It is not known why stuttering is more common in males; however, it is thought that this may be associated with prenatal testosterone levels. ${ }^{4,5}$

Even though the etiology of developmental stuttering, the most frequent type, has not yet been elucidated, it has been associated with various factors such as atypical neurophysiology, a motor dysfunction in speech, cognitive processing abilities, genetics and environmental impacts. ${ }^{6,7}$ Several researchers state that neurological factors play an important role in the etiology of stuttering. ${ }^{8}$ Numerous studies reported in the literature support a relationship between stuttering and atypical cerebral lateralization. ${ }^{7}$ Variations in hemispheric lateralization are thought to be associated with varying prenatal testosterone exposure. The Geschwind Behan Galaburda (GBG) hypothesis 9,10 and the callosal hypothesis ${ }^{11,12}$ suggest that among 
the androgens, prenatal testosterone causes atypical cerebral lateralization and this atypical cerebral lateralization may result in problems related to language development and skills. ${ }^{13}$

The aim of this study was to examine the relationship between prenatal testosterone and developmental stuttering in boys. With stuttering considered to be a speech disorder occurring largely in the three to eight years' age range, the question of how to evaluate prenatal testosterone during the postnatal period in order to examine the relationship between stuttering and prenatal testosterone, presents a challenge.

It has been suggested that from the late first trimester, the ratio of the length of the $2^{\text {nd }}$ digit (index finger) to the $4^{\text {th }}$ digit (ring finger) (2D:4D) has a negative correlation with prenatal testosterone levels and a positive correlation with prenatal estrogen levels. ${ }^{14}$ Studies on rats have revealed that prenatal testosterone elevated by genetic and hormonal manipulation will stimulate the growth of the fourth fetal phalanx. ${ }^{15,16}$ Additionally, growth of the adrenal gland has been found to increase prenatal androgen levels and cause a low 2D:4D ratio in children with congenital adrenal hyperplasia. ${ }^{17}$ 2D:4D ratio has been used to evaluate the correlation between prenatal exposure to testosterone and some neurodevelopmental conditions such as autism. ${ }^{18,19}$ Within the literature covering this topic, it can be suggested that the $2 \mathrm{D}: 4 \mathrm{D}$ ratio is a marker for testosterone exposure in the prenatal period. In the present study, the right and left hand 2D:4D ratios were compared between boys who stutter and boys who do not stutter.

In considering the possible effects of prenatal testosterone on hemispheric lateralization and the possibility that atypical hemispheric lateralization plays an important role in the etiology of developmental stuttering, the hypothesis of this study was that prenatal testosterone plays a role in the etiology of developmental stuttering. In the literature, we found only two studies that investigated the relationship between prenatal testosterone and stuttering. The first study, by Montag et al. ${ }^{20}$, was conducted with adults and there was no significant relationship between prenatal testosterone (2D:4D) and stuttering. The second study, by Mohammadi et al. ${ }^{21}$, was conducted with children. Also there was no significant relationship between prenatal testosterone (2D:4D) and stuttering. The current study was conducted with child patients as childhood is the period when stuttering most frequently occurs, therefore this study is considered to be the second in the literature.

\section{Material and Methods}

\section{Population}

This cross-sectional study was conducted in the child psychiatry clinics of Inonu University and Necmettin Erbakan University Medical Faculty Hospital. Approval for the study was obtained from the Malatya Clinical Trials Ethics Committee (Ethics Committee decision number: 2015/93, date Jun 17, 2015). The aim and methods of the research were explained in detail to the boys and their families, and after signing consent forms they were included in the study. In total, 133 boys (49 boys who stutter and 84 boys who do not stutter) were included. The age range of children participating in the study was determined as 2-12 years. The stuttering group consisted of boys who had applied to the child psychiatry clinics due to problems with stuttering and who were subsequently diagnosed with developmental stuttering. The control group consisted of completely healthy boys. Stuttering is more common in males than in females and gender was thought to be a confounding factor, so only boys were included in the study. In both groups, individuals with a family history of stuttering were not included in the study in order to prevent genetic factors from affecting the outcome of the study. Additionally, individuals with mental retardation, neurological disease or comorbid chronic disease, a history of head or hand trauma were not included.

\section{Evaluation and measurements}

Diagnosis of developmental stuttering was established by child psychiatrists based on diagnostic criteria from the Diagnostic and Statistical Manual of Mental Disorders, 4th ed., Text Revision (DSM-IVTR). ${ }^{22}$ Duration and severity of stuttering of individuals with stuttering were evaluated. Clinical Global Impression-Severity Scale (CGI-S) was used in the evaluation of severity of stuttering. CGI-S is a 7 -point single-item scale $(0=$ no evaluation; $1=$ normal, no illnesses; 2 =borderline; 3 =mild; 
Table I. The Duration of Stuttering and the Severity of Stuttering of Individuals with Stuttering $(\mathrm{N}=49)$.

\begin{tabular}{lc}
\hline Duration of stuttering & N (\%) \\
\hline $0-6$ months & $7(14.3)$ \\
$7-12$ months & $7(14.3)$ \\
$13-24$ months & $6(12.2)$ \\
$25-36$ months & $6(12.2)$ \\
$>36$ months & $23(46.9)$ \\
Severity of stuttering & $3.511 .44 \pm$ (range: $1-7)$
\end{tabular}

Clinical Global Impression-Severity Scale (CGI-S) was used to evaluate the severity of stuttering.

$4=$ moderate; $5=$ prominent; $6=$ severe; $7=$ most severe), used by investigators in order to record either illness severity. ${ }^{23}$

The lengths of the $2^{\text {nd }}$ and $4^{\text {th }}$ digits of both hands were measured from the ventral proximal line. Measurements were performed by the authors (Dönmez YE and Bilgiç A) using a digital vernier caliper with $0.01 \mathrm{~mm}$ precision. The ratio of the $2^{\text {nd }}$ digit to the $4^{\text {th }}$ digit in both hands was calculated as right 2D:4D and left 2D:4D. The stuttering group and the control group were compared in terms of right and left 2D:4D.

\section{Statistical analysis}

The Statistical Package for the Social Sciences (SPSS) 22.0 program was used in the study. The results of Shapiro-Wilk normality analysis showed that the data of age showed a normal distribution and an independent sample t-test was used. But the data of right and left 2D:4D ratios was not normally distributed therefore a Mann-Whitney $U$ test was used in the comparison of the groups. $\mathrm{P}$ values less than 0.05 were considered statistically significant.

\section{Results}

The mean age of the stuttering group was 7.062.33 \pm years (range: $2-12$ years) and control group was 6.792.36 \pm years (range: 2-12 years).
There was no statistically significant difference between the groups in terms of age $(p=0.487)$. The descriptive data of duration and severity of stuttering of individuals with stuttering are shown in Table I.

In the comparative evaluation conducted between the stuttering group and the control group in terms of their right $2 \mathrm{D}: 4 \mathrm{D}$ and left 2D:4D ratios, it was found that the left 2D:4D ratio of the patients was significantly higher than that of the control group $(p=0.017)$. However, there was no significant statistical difference between the groups in terms of right $2 \mathrm{D}: 4 \mathrm{D}$ ratios $(\mathrm{p}=0.595$; Table II).

\section{Discussion}

When the results of the present study were examined, a significant difference was found between the stuttering group and control group in terms of left 2D:4D ratios. The left 2D:4D ratio of the stuttering group was significantly higher than that of the control group. This result indicates that patients had experienced low prenatal testosterone exposure, and this suggests that prenatal testosterone may play a role in the etiology of developmental stuttering.

Only two studies have been found in the literature that examined the relationship between prenatal testosterone and stuttering. In the first study by Montag et al. ${ }^{20}$, the sample

Table II. Comparison of Digit Ratio Measurements.

\begin{tabular}{lcccc}
\hline & \multicolumn{3}{c}{ Percentiles } & \multirow{2}{*}{ p value } \\
\cline { 2 - 4 } & 25th & 50th (median) & 75th & \\
\hline Right 2D:4D & 1.0069 & 1.0331 & 1.0545 & 0.595 \\
Left 2D:4D & 1.0049 & 1.0253 & 1.0487 & $0.017^{*}$ \\
\hline
\end{tabular}

2D:4D: the ratio of the length of the $2^{\text {nd }}$ digit (index finger) to the $4^{\text {th }}$ digit (ring finger) 
consisted of adult patients and no significant correlation between stuttering and prenatal testosterone (2D:4D) was identified. However, they reported a negative correlation between the left digit ratio and negative experiences of people who stuttered, using the Overall Assessment of the Speaker's Experience of Stuttering (OASES), suggesting that higher prenatal testosterone is linked to more negative experiences due to stuttering. The second study, by Mohammadi et al. ${ }^{21}$, was conducted with children. Once again, there was no significant relationship between prenatal testosterone (2D:4D) and stuttering. But they showed that postnatal serum levels of testosterone and its metabolites, dihydrotestosterone and oestradiol, were significantly higher in child who stuttered compared to child who did not stutter. They also found a significant positive correlation between postnatal testosterone, dehydroepiandrosterone and cortisol levels and stuttering severity. In the present study, there were no evaluations and comparisons in terms of severity of stuttering. Severity and duration of stuttering were given to know patients' stuttering characteristics. The results of our study are different from these two studies, which were done using similar methods (by the use of $2 \mathrm{D}: 4 \mathrm{D}$ ratio). For this reason, our study is the first study in the literature to reveal the relationship between prenatal testosterone (2D:4D) and developmental stuttering.

In the literature review carried out to clarify the role of prenatal testosterone in the etiology of developmental stuttering, various pathophysiological mechanisms were identified that could explain this relationship. Although these mechanisms do not directly explain the relationship between prenatal testosterone and developmental stuttering, it can be possible to explain this pathophysiology in two steps by establishing a cause-effect relationship. While the first step explains the relationship between prenatal testosterone exposure and atypical hemispheric lateralization, the second step explains the relationship between atypical hemispheric lateralization and developmental stuttering.

The GBG hypothesis and the callosal hypothesis, both of which are important theories, endeavor to explain the relationship between prenatal testosterone exposure and hemispheric lateralization. ${ }^{13}$ The hypotheses differ in terms of the biological mechanism used to describe the relationship. The GBG hypothesis suggests that prenatal testosterone slows down the growth of some cortical areas in the left hemisphere during the prenatal period, which is a critical time in terms of neurodevelopment. ${ }^{9}$ High levels of testosterone lead to the right hemisphere being dominant in relation to functions such as language skills through a disruption of the development of the left hemisphere.

On the other hand, the callosal hypothesis suggests that low prenatal testosterone decreases axonal pruning in the corpus callosum and this leads to atypical lateralization by affecting the flow of information between the hemispheres. ${ }^{11,12}$ The corpus callosum is the main pathway between the two hemispheres and axonal pruning in this area increases hemispheric specialization related to various functions. ${ }^{24}$ Axonal pruning is thought to improve functional circuits by selectively eliminating connections between neurons and synapses and thus causing more efficient brain functions. ${ }^{25}$ However, in the case of low prenatal testosterone exposure, it is suggested that sufficient axonal pruning does not occur in the corpus callosum, resulting in problems in hemispheric specialization.

When comparing these two hypotheses, it can be concluded that the results of the present study support the callosal hypothesis. The left 2D:4D ratio of the stuttering group was significantly higher than the control group and this indicated that the stutterers were exposed to less prenatal testosterone.

There are various studies in the literature that support the relationship between prenatal testosterone and atypical hemispheric lateralization that fall within the ambit of the callosal hypothesis. Hollier et al. ${ }^{26}$ report that a low umbilical testosterone level is associated with atypical hemispheric lateralization related to language. Studies by Lust et al. ${ }^{27}$ and Grimshaw et al. ${ }^{28}$ examining language based functions both reveal that a high amniotic testosterone level is associated with left hemisphere dominance in terms of language, in other words, high prenatal testosterone accompanies typical hemispheric lateralization.

Several researchers reported in the literature 
support a relationship between atypical cerebral lateralization and stuttering. Neuroimaging studies conducted with adults reveal that individuals who stutter have a variety of structural and functional abnormalities relative to healthy individuals. ${ }^{29-33}$ In studies on speech production, when individuals with stuttering are compared with individuals speaking fluently, excess activation is observed in the right hemisphere of the stuttering individuals but decreased activation is observed in their left superior temporal, fronto-temporal and temporo-parietal areas. The studies on the relationship between atypical hemispheric lateralization and stuttering have mainly been conducted on adult patients. In a limited number of studies conducted with children, the relationship between atypical hemispheric lateralization and developmental stuttering was reported. ${ }^{31,34-37}$ In the study by Sato et al. ${ }^{7}$ with three to five year-old children, abnormal hemispheric lateralization related to receptive speech processing in the posterior language area was present in those children with stuttering.

The primary aim of the present study was to determine the relationship between prenatal testosterone and developmental stuttering. Hemispheric lateralization was not evaluated. We have mentioned information in the literature linking prenatal testosterone with atypical hemispheric lateralization and atypical hemispheric lateralization with stuttering for the purpose of bringing a pathophysiological explanation to the relationship we identified between prenatal testosterone and stuttering. The fact that hemispheric lateralization was not evaluated in the present study could be considered a limitation. The hand dominance of the children participating in the study could have been included in the evaluation in order to provide an indication of cerebral lateralization. However, studies have shown that $97 \%$ of individuals with right hand dominance have left hemisphere lateralization in terms of language, and $3 \%$ have bilateral or right hemisphere lateralization; while $60 \%$ of individuals with left hand dominance have left hemisphere lateralization, $30 \%$ have bilateral lateralization, and $10 \%$ have right hemisphere lateralization. ${ }^{38}$ When this information was considered, it was decided that hand dominance would not yield correct or significant results in evaluating cerebral lateralization. In addition, neuroimaging methods for direct measurement of cerebral lateralization were not used in the present study due to the researchers' concerns about security and adaptation problems (such as waiting inactively for a long time) since the study was to be conducted with children.

When considering the method to be used in the present study for a determination of prenatal testosterone exposure, it was decided to measure the $2 \mathrm{D}: 4 \mathrm{D}$ ratio directly by using a digital vernier caliper with $0.01 \mathrm{~mm}$ precision. Although more difficult to obtain, direct digit measurements are accepted as being more reliable than indirect methods (scanning, photocopying). ${ }^{39}$

It was observed that different methods were used in the studies assessing prenatal testosterone but not related to stuttering. These methods included measurement of amniotic testosterone concentration 40 and umbilical cord testosterone concentration. ${ }^{41}$ Amniotic testosterone concentration and umbilical cord testosterone concentration can both give clearer and more accurate results than the $2 \mathrm{D}: 4 \mathrm{D}$ ratio. However, because developmental stuttering develops mainly in the age range of three to eight years, and developmental stuttering was the topic under investigation in the present study, it seemed that the 2D:4D ratio, which is an accepted marker for the evaluation of prenatal testosterone in the postnatal period, was the most logical method to use. But, some researchers have suggested that $2 \mathrm{D}: 4 \mathrm{D}$ ratio is not a reliable indicator of prenatal testosterone in the postnatal period. ${ }^{42}$

Another limitation of the present study is that it was a cross-sectional study and the number of the sample was limited. Only boys were included in the study to prevent gender from becoming a confounding factor. Nevertheless, this may be a limitation of the study.

Consequently, as the authors, we are of the opinion that prenatal testosterone may play a role in the etiology of developmental stuttering in boys. However, it will be necessary to conduct further studies evaluating both prenatal testosterone and hemispheric lateralization in order to clarify the pathophysiological mechanisms more explicitly. 


\section{REFERENCES}

1. Gordon N. Stuttering: incidence and causes. Dev Med Child Neurol 2002; 44: 278-282.

2. Ludlow CL. Stuttering: dysfunction in a complex and dynamic system. Brain 2000; 123: 1983-1984.

3. Craig A, Hancock K, Tran Y, Craig M, Peters K. Epidemiology of stuttering in the community across the entire life span. J Speech Lang Hear Res 2002; 45: 1097-1105.

4. Selçuk EB, Gönenir Erbay L, Özel Özcan Ö, Kartalci Ş, Batcioğlu K. Testosterone levels of children with a diagnosis of developmental stuttering. Ther Clin Risk Manag 2015; 11: 793-798.

5. Bloodstein O, Ratner NB. A Handbook on Stuttering (6th ed). Clifton Park, NY: Thomson Delmar, 2008.

6. Prasse JE, Kikano GE. Stuttering: an overview. Am Fam Physician 2008; 77: 1271-1276.

7. Sato Y, Mori K, Koizumi T, et al. Functional lateralization of speech processing in adults and children who stutter. Front Psychol 2011; 27: 70.

8. Max L, Guenther FH, Gracco VL, Ghosh SS, Wallace ME. Unstable or insufficiently activated internal models and feedback-biased motor control as sources of dysfluency: A theoretical model of stuttering. Contemp Issues Commun Sci Disord 2004; 31: 105-122.

9. Geschwind N, Galaburda AM. Cerebral lateralization: Biological mechanisms, associations, and pathology: I. A hypothesis and a program for research. Arch Neurol 1985; 42: 428-459.

10. Hollier L. Fetal testosterone exposure and its relationship to the development of language and cerebral laterality (Doctoral dissertation). University of Western Australia: School of Psychology and Telethon Kids Institute, 2014

11. Witelson SF. Neural sexual mosaicism: Sexual differentiation of the human temporo-parietal region for functional asymmetry. Psychoneuroendocrinology 1991; 16: 131-153.

12. Witelson SF, Nowakowski RS. Left out axoms make men right: A hypothesis for the origin of handedness and functional asymmetry. Neuropsychologia 1991; 29: 327-333.

13. Cohen-Bendahan CC, Buitelaar JK, van Goozen SH, Cohen-Kettenis PT. Prenatal exposure to testosterone and functional cerebral lateralization: A study in same-sex and opposite-sex twin girls. Psychoneuroendocrinology 2004; 297: 911-916.

14. Manning JT. Digit ratio. A pointer to fertility, behaviour, and health. New Brunswick, NJ: Rutgers University Press, 2002.

15. Manning JT. Resolving the role of prenatal sex steroids in the development of digit ratio. Proc Natl Acad Sci USA 2011; 108: 16143-16144.

16. Zheng Z, Cohn MJ. Developmental basis of sexually dimorphic digit ratios. Proc Natl Acad Sci USA 2011; 108: 16289-16294.

17. Hönekopp J, Watson S. Meta-analysis of digit ratio 2D: $4 \mathrm{D}$ shows greater sex difference in the right hand. Am J Hum Biol 2010; 22: 619-630.
18. Al-Zaid FS, Alhader AA, Al-Ayadhi LY. The second to fourth digit ratio (2D:4D) in Saudi boys with autism: A potential screening tool. Early Hum Dev 2015; 91: 413-415.

19. Guyatt AL, Heron J, Knight Ble C, Golding J, Rai D. Digit ratio and autism spectrum disorders in the Avon Longitudinal Study of Parents and Children: A birth cohort study. BMJ Open 2015; 5: e007433.

20. Montag C, Bleek B, Breuer S, et al. Prenatal testosterone and stuttering. Early Hum Dev 2015; 91: 43-46.

21. Mohammadi H, Joghataei MT, Rahimi Z, et al. Sex steroid hormones and sex hormone binding globulin levels, CYP17 MSP AI (-34T:C) and CYP19 codon 39 (Trp:Arg) variants in children with developmental stuttering. Brain Lang 2017; 175: 47-56.

22. American Psychiatric Association. Diagnostic and Statistical Manual of Mental Disorders, (4th ed), Text Revision (DSM-IV-TR). Washington, DC: 2000.

23. Guy W. ECDEU Assessment Manual for Psychopharmacology, Revised. US Department of Health, Education, and Welfare Publication (ADM) Rockville, MD: National Institute of Mental Health, 1976: $76-338$

24. Häberling IS, Badzakova-Trajkov G, Corballis MC. Callosal tracts and patterns of hemispheric dominance: a combined fMRI and DTI study. Neuroimage 2011; 54: 779-786.

25. Low LK, Cheng HJ. Axon pruning: an essential step underlying the developmental plasticity of neuronal connections. Philos Trans R Soc Lond B Biol Sci 2006; 361: 1531-1544.

26. Hollier LP, Maybery MT, Keelan JA, Hickey M, Whitehouse AJ. Perinatal testosteron eexposure and cerebral lateralisation in adult males: evidence for the callosal hypothesis. Biol Psychol 2014; 103: 48-53.

27. Lust JM, Geuze RH, Van de Beek C, Cohen-Kettenis PT, Groothuis AGG, Bouma A. Sex specific effect of prenatal testosterone on language lateralization in children. Neuropsychologia 2010; 48: 536-540.

28. Grimshaw GM, Bryden MP, Finegan JAK. Relations between prenatal testosterone and cerebral lateralization in children. Neuropsychology 1995; 9: 68-79.

29. Beal DS, Cheyne DO, Gracco VL, Quraan MA, Taylor MJ, De Nil LF. Auditory evoked fields to vocalization during passive listening and active generation in adults who stutter. Neuroimage 2010; 52: 1645-1653.

30. Kaganovich N, Wray AH, Weber-Fox C. Non-linguistic auditory processing and working memory update in pre-school children who stutter: an electrophysiological study. Dev Neuropsychol 2010; 35: 712-736.

31. Liotti M, Ingham JC, Takai O, Paskos DK, Perez R, Ingham RJ. Spatiotemporal dynamics of speech sound perception in chronic developmental stuttering. Brain Lang 2010; 115: 141-147.

32. Lu C, Peng D, Chen C, et al. Altered effective connectivity and anomalous anatomy in the basal ganglia-thalamocortical circuit of stuttering speakers. Cortex 2010; 46: 49-67. 
33. Kikuchi Y, Ogata K, Umesaki T, et al. Spatiotemporal signatures of an abnormal auditory system in stuttering. Neuroimage 2011; 55: 891-899.

34. Özge A, Toros F, Çömelekoğlu Ü. The role of hemispheral asymmetry and regional activity of quantitative EEG in children with stuttering. Child Psychiatry Hum Dev 2004; 34: 269-280.

35. Chang SE, Erickson KI, Ambrose NG, HasegawaJohnson MA, Ludlow CL. Brain anatomy differences in childhood stuttering. Neuroimage 2008; 39: 1333-1344.

36. Weber-Fox C, Spruill III JE, Spencer R, Smith A. Atypical neural functions underlying phonological processing and silent rehearsal in children who stutter. Dev Sci 2008; 11: 321-337.

37. Beal DS, Quraan MA, Cheyne DO, Taylor MJ, Gracco VL, De Nil LF. Speech-induced suppression of evoked auditory fields in children who stutter. Neuroimage 2011; 54: 2994-3003

38. Ypsilanti A, Ganou M, Koidou I, Grouios G. Digit ratio (2D: 4D) in individuals with intellectual disability: Investigating the role of testosterone in the establishment of cerebral lateralisation. Laterality 2008; 13: 527-544.
39. Whitehouse AJO, Mattes E, Maybery MT, et al. Sex-specific associations between umbilical cord blood testosterone levels and language delay in early childhood. J Child Psychol Psychiatry 2012; 53: 726-734

40. Brown WM, Hines M, Fane BA, Breedlove SM Masculinized finger length patterns in human males and females with congenital adrenal hyperplasia. Horm Behav 2002; 42: 380-386.

41. Ökten A, Kalyoncu M, Yaris N. The ratio of second- and fourth-digit lengths and congenital adrenal hyperplasia due to 21-hydroxylase deficiency. Early Hum Dev 2002; 70: 47-54

42. Hollier LP, Keelan JA, Jamnadass ESL, Maybery MT, Hickey M, Whitehouse AJO. Adult digit ratio (2D:4D) is not related to umbilical cord androgen or estrogen concentrations, their ratios or net bioactivity. Early Hum Dev 2015; 91: 111-117. 